\title{
An Efficient Framework for Probabilistic Search with Multi-UAVs
}

\author{
Shaohua Qin ${ }^{1, a, *}$, Xianfeng $\mathbf{L i}^{1, b}$ \\ ${ }^{1}$ School of Electronic and Computer Engineering, Peking University, Shenzhen, China \\ aqinshaohua@sz.pku.edu.cn, blixianfeng@pkusz.edu.cn \\ *Corresponding author
}

Keywords: Multi-UAVs, Data fusion, Probabilistic search

\begin{abstract}
This paper focuses on the task of searching a stationary target using a team of multiple unmanned aerial vehicles (UAV) with limited communication ranges and sensing capabilities. It is based on a probabilistic representation model of the search environment, in which each UAV keeps a probability map on the presence of the target for each cell. The UAVs make observations and update the map for multiple rounds according to the Bayesian rule. In this process, each UAV can exchange data with its neighbors who are in its communication range. The decision of finding the target can be made once its probability of presence in any cell reaches a threshold. In this work, we design an efficient framework to decide the movement of each UAV at each time step, and propose a novel data fusion strategy among UAVs. The simulation result shows that our framework achieves improvements on both the success rate and the time taken to complete the searching task. We also explore the impact of different parameters, like the sensor model, moving mobility model of each UAV, and the data fusion strategies associated with the moving patterns. The simulation results show that the correct rate on target identification can vary greatly under different parameter settings, indicating the importance of the selection of UAV searching components such as the sensor model and the mobility model.
\end{abstract}

\section{Introduction}

Unmanned aerial vehicles (UAVs) are being widely used in wilderness search areas [1],[2]. For each UAV, its task is to gather enough information to determine whether the target is in the search area, and to report the correct location of the target. Searching the target in a wide area with multiple UAVs as a collaborative team can certainly speed up the process of finding the target, and a searching team can also increase the credibility of the searching result affected by probabilitybased observations on the presence of the target [3].

In the team-based searching, the search area is decomposed into a number of cells. Each UAV contains a map of the search area, which reflects the probabilities on the presence of the target in various cells. The map is initialized with neutral probability $(0.5)$ for each cell. With the movement of the UAVs and their observations, the search map gets updated gradually. During this course, UAVs may communicate with others to decide what to do next. We treat a UAV with there parts: (1) a sensor to identify its current cell, and an observation to update the cell and the search map, (2) a 
wireless transceiver to communicate with others within its communication range to fusion information, such as its probability map, its observation and a time stamp matrix, (3) a decision maker to decide where to move and whether to finish the search [4].

Our goal is to find the target as soon as possible with high confidence. Although there are some researchers focus on this problem [5], few of them treat the searching step and decision making step together [6],[7]. Although some people treat them as an integral problem, there are some shortcomings with them. First, the UAV uses the pre-planned trajectories, which means that the UAVs are not autonomous; instead, the sensor operators take the responsibility. Second, the communication range limits the ability of information exchange between UAVs, which may also have impacts on the final results, making it an error-prone process. Finally, the human resources to operate the UAVs can be insufficient [8].

Our way to mitigate the problems above is to highly automate the UAVs and develop high efficient data fusion strategies. Our major contribution in this paper is to propose a framework which treats the searching task and UAV's movement online together and which contains some efficient data fusion strategies in probabilistic search and moving strategy in real-time step by step. In other words each UAV can decide its movement depending on the observations or the probability map online, so that each UAV can change its pre-planned trajectories to achieve better results.

The paper is organized as follows. Section 2 discusses the related work in UAV searching for a target in unknown environment. In Section 3 presents the environment model, the UAV probabilistic model and the searching strategy. Section 4 describes our framework and some data fusion strategies and the UAV's movement online step by step. In Section 5, results are presented and discussed. Finally, we concludes the paper and our future work.

\section{Related Work}

The problem of searching has been an intensive research. We can classify existing search research into three kinds. The first kind focuses on efficiently search the whole region, and the second concentrates on data fusion, and the third approach is about the movement of each UAV[9],[10]. The difference between them is the data they exchange with others and when or whom to share their information. Under different scenes, the model can be largely different, what we focus is the scene described in section 1. So the search in the last two kinds is in our attention [11],[12].

The basic components in the search problem include a prior distribution on target location, a function associated with the detection probability. We finish the search mission when the probability in any cell is beyond a threshold. Each UAV has a sensor model which will be introduced in section 3. The target's existing probability is updated by a Bayesian rule and its observation. There have different data fusion strategies, but most of them assume the UAVs can communicate with each other all the time.

About the movement, a large number researches have a pre-defined assumption that the UAV can not re-planned its movement no matter how the UAVs are distributed [13],[14],[15]. And most of them is associated with the first kind of search such as lane base search, random search, grid based search [16]. Their purpose is to reduce the uncertainty about the target existence probability in its current cell and to avoid covering the same search area. But the information they get is from the flight base, which may be not enough [17]. So in our paper we present our framework to let the UAVs can decide where to go online step by step.

So in this paper we treat the data fusion step and the movement of each UAV as a whole problem. We introduce a framework the UAV can decide its movement online with limited communication and sensing capabilities efficiently. 


\section{Problem Statement}

We model the problem as follows: The UAVs are searching for a signal stationary target in a rectangular area, which is decomposed into $\mathrm{L} \cdot \mathrm{M}$ cells ( $\mathrm{L}$ is the length and $\mathrm{M}$ is the width). So the search area is represented as $\Omega=\{1,2,3 \ldots \mathrm{L} \cdot \mathrm{M}\}$. And the target or the UAV is at most in and only in one of the cells. There is at most one target denoted as T in search area $\Omega$.

The target's occupancy probability is modelled as a Bernoulli Distribution [18], that is $X_{c}=1$ (target is in cell c) as probability $\mathrm{P}_{\mathrm{c}}$ and target is not in cell in $\mathrm{c}\left(\mathrm{X}_{\mathrm{c}}=0\right)$ is probability $1-\mathrm{P}_{\mathrm{c}}$. If cell $\mathrm{c}$ have not been searched, $\mathrm{Pc}=0.5$. We finish the search if any cell's probability is above the predefined threshold $\mathrm{B}_{\text {up }}$. If $\mathrm{P}_{\mathrm{c}}<\mathrm{B}_{\text {down }}$, we treat cell $\mathrm{c}$ as an empty cell, which will no longer be searched by any UAV in the future. If all the cells are empty we also finish the search.

There are a number of homogeneous UAVs $U=\left\{\mathrm{U}_{1}, \mathrm{U}_{2} \ldots \mathrm{U}_{\mathrm{N}}\right\}$ doing the search work, and each UAV is equipped with : (1) a sensor that can sense its current position and its observation about the current searching cell, (2) an information fusion component that let it can exchange data with others, (3) a decision maker to decide what to do next.

We assume each UAV can move to its left, right, forward, backward cell or stay in its current cell. The UAV decides where to move at every time step depending on its probability map. We assume that only one observation per cell can be taken at a single time step. $Q_{i, c, t}$ denotes the observation result of UAV $\mathrm{U}_{\mathrm{i}}$ on cell $\mathrm{c}$ at time $\mathrm{t}$. The observation result can be $Q_{i, c, t}=0$ (negative observation) or $Q_{i, c, t}=1$ (positive observation). We can formally express our sensor model as follows:

$$
\begin{aligned}
& P\left(Q_{i, c, t}=1 \mid \mathrm{X}_{c}=1\right)=p \\
& P\left(Q_{i, c, t}=1 \mid \mathrm{X}_{c}=0\right)=q \\
& P\left(Q_{i, c, t}=0 \mid \mathrm{X}_{c}=1\right)=1-p \\
& P\left(Q_{i, c, t}=0 \mid \mathrm{X}_{c}=0\right)=1-q
\end{aligned}
$$

The $\mathrm{p}$ and $\mathrm{q}$ represent the target detection probability and false alarm probability, normally $0<q<0.5<p<1$. As for communication range, we assume that the communication ranges among UAVs are measured by their Euclidean distance, which is limited to $r$ cells. We also assume the probability of a cell $\mathrm{C}_{\mathrm{i}}$ can not affect the existing probability of other cells. Thus, the information can only be exchanged when the UAVs are within distance $r$.

Let $P_{i, c, t}$ represent the probability of $\mathrm{UAV} \mathrm{U}_{\mathrm{i}}$ at time $\mathrm{t}$ about the cell $\mathrm{C}$, and the $P_{i, c, t}$ for all cells is the search map of UAV $U_{\mathrm{i}}$ at time t. Each UAV updates its search map according its observation based on the following expression.

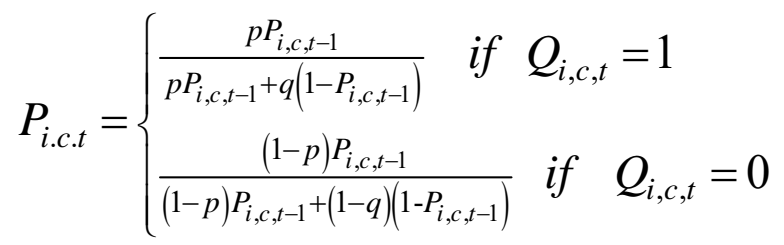

Since the observation only gets probabilistic results, there exists chances that we get wrong search result, where an empty cell is taken as the location for the target. Data fusion of UAVs can help reduce the chances for wrong search results, which we will introduce in the next section. 


\section{Data fusion and Real-time Movement}

We have introduced a framework for the system model described in Section 3, in which the working process of a UAV is shown in Figure 1. Each UAV first senses its location and takes one time observation to its current cell $\mathrm{C}_{\mathrm{i}}$, and updates its own search map according to the Bayesian rule described in Section 3. Next the UAV can receive the $C_{i}$ information of other UAVs, and update their probability about cell $\mathrm{C}_{\mathrm{i}}$ accordingly. If any UAV finds a probability above $\mathrm{B}_{\text {up }}$, the search is completed, and the cell with its probability above $\mathrm{B}_{\mathrm{up}}$ is treated as the location for the target.

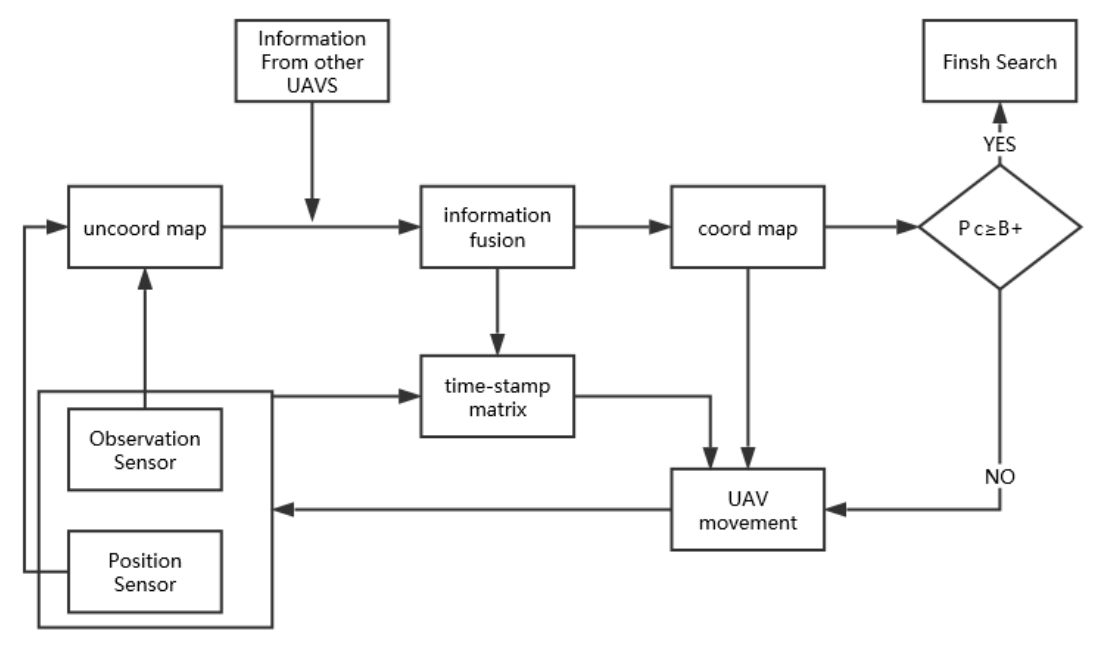

Figure 1 System Framework

In the information fusion step, UAVs work together to get enough information to guide decisionmaking for their next step, as shown in Figure 3. Here we introduce some data fusion strategies:

The first one is the belief update strategy. In this strategy, each UAV updates its search map independently without exchanging any data with each other. In essence, $U A V U_{i}$ at cell $C_{j}$ computes the uncoordinated occupancy probability for cell $\mathrm{C}_{\mathrm{j}}$, and broadcasts it to others. UAVs receiving this update will overwrite their previous values at cell $C_{j}$ with the broadcasting of $U_{i}$. We assume $U_{k}$ receives the update information of $U_{i}$ about $C_{j}$, this can be expressed as follows:

$$
P_{\mathrm{k}, \mathrm{C}_{j}}=P_{\mathrm{i}, \mathrm{C}_{j}}
$$

The second one is the average strategy in which each UAV averages the information it gets from others. If $U_{i}$ receives the information from others about $C_{j}$, and if $U_{j}$ has observed cell $C_{j}$, we compute the average of all $U_{j}$ that are within the communication range of $U_{i}$. This can be expressed as follows, $n$ is the number of UAVs that $U_{i}$ can exchange data with.

$$
P_{\mathrm{k}, \mathrm{C}_{j}}=\frac{1}{n} \sum_{i=1}^{n} P_{\mathrm{i}, \mathrm{C}_{j}} \quad(\mathrm{i} !=\mathrm{k})
$$

This strategy above may face a problem. For the same cell, the time that UAVs visiting it may have big difference. The recently visited UAV have the newest information, so we propose the third strategy called time-stamp method, which considers the time-stamp matrix R denoting the search time of each cell. This matrix can be expressed as follows: $n$ is the number of UAVs within $U_{k}$ 's communication range, and $t_{\min }$ is the oldest time among all of the $n$ UAVs observing cell $C_{j}$. This 
method can ensure that the UAV which has newer information can contribute more to our search than the others.

$$
P_{\mathrm{k}, \mathrm{C}_{j}}=\sum_{i=1}^{n} \frac{\mathrm{t}_{\mathrm{i}}+1-\mathrm{t}_{\mathrm{min}}}{\sum_{i=1}^{n}\left(\mathrm{t}_{\mathrm{i}}+1-\mathrm{t}_{\mathrm{min}}\right)} P_{\mathrm{i}, \mathrm{C}_{j}}
$$

In this strategy, UAV's decision on its next move will be affected by its searching history around the current cell (left, right, behind and back). It will search the cell that has the highest probability.

\section{Simulation Results}

We simulate our framework on a $10 \times 10$ search area, and the target is located in cell $(6,7)$ position, we use four homogeneous UAVs to do the search work. To study the impact of the parameters on the search quality (speed and correctness), the sensor quality varies with $p=0.1,0.2$, $0.3,0.4$ and $\mathrm{q}=0.8,0.9$; and the communication ranges varies with $2,4,6,8,10,15$, and 20 cells respectively. Initially, four UAVs are located in the four corners of our search area. Their moving rules are described in Section 4.

There are three sets of experiments: (1) uncoordinated map search, where each UAV search independently; (2) Search with unlimited communication range, where information can be exchanged among all UAVs at any time; and (3) Search with limited communication range search (a more realistic setting compared to the previous one). We implement the framework in MATLAB, and the experiment is repeated for 1000 times to increase the robustness of the experiment. The results are presented as follows:

First, we can see from Table 1 that $p$ has a great impact on the search time, because $p$ is the correct detection probability. On the other hand, q has a large impact on the rate of correctness, because $\mathrm{q}$ is the false alarm rate.

Second, we analyze the impact of the communication range $r$ under different data fusion strategies. The results are shown in Figure 2. We can see from that our strategy achieves very high rate of correctness with reasonable communication ranges. We also find that the search time is closely related to its mobility model. For example, the search time of random movement can be 100 time steps much more than the moving rules of our framework. When using the pre-planned path, it can be 200 time steps more.

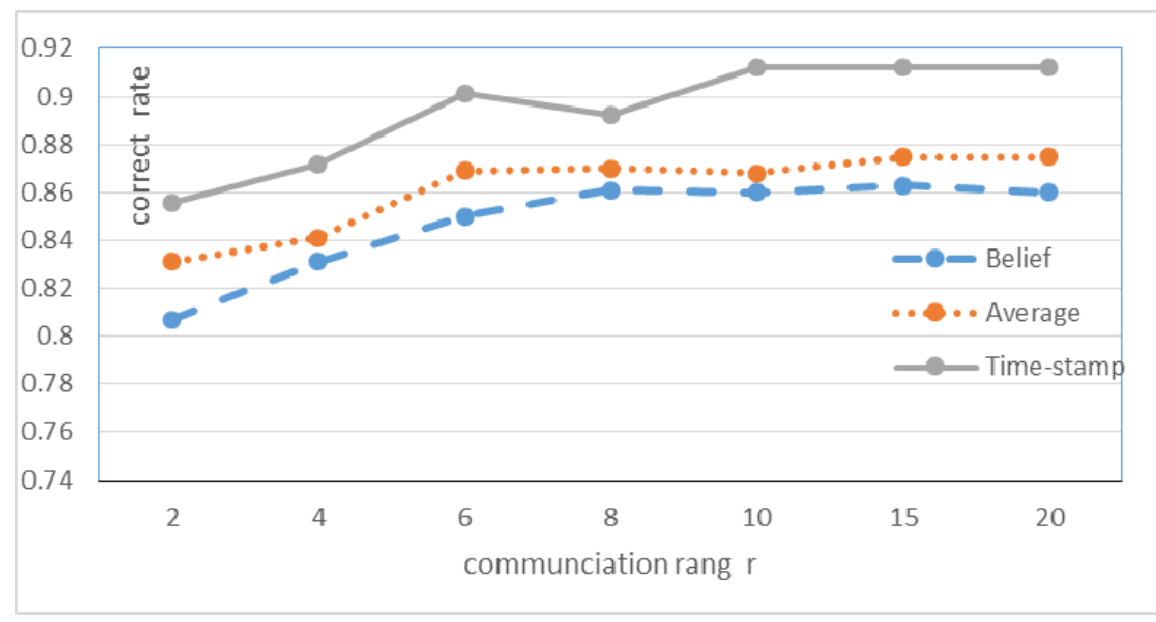

Figure 2 The impact of data fusion strategies and communication range on correct rate 
Table 1: The influence of $\mathrm{p}$ and $\mathrm{q}$

\begin{tabular}{|l|l|l|l|}
\hline $\mathrm{p}$ & $\mathrm{q}$ & Search time & Success Rate \\
\hline 0.9 & 0.1 & 31.2390 & 0.9100 \\
\hline 0.9 & 0.2 & 36.0790 & 0.8190 \\
\hline 0.9 & 0.4 & 43.3900 & 0.6053 \\
\hline 0.8 & 0.1 & 31.1820 & 0.8920 \\
\hline 0.8 & 0.2 & 45.2100 & 0.8130 \\
\hline 0.8 & 0.4 & 66.4690 & 0.6010 \\
\hline
\end{tabular}

\section{Conclusions}

Searching targets using a team of UAVs is a promising yet challenging problem. In this paper, we treat data fusion strategy among UAVs and the moving rules of individual UAVs as a whole problem, and propose a framework for this problem. From the result in Section 5, the rate of correctness is closely related to $q$ of the sensor quality, the communication range and the data fusion strategy. The search time is largely influenced by the $\mathrm{p}$ and the mobility rules of UAVs. The higher the $\mathrm{p}$ is, the lower our search time is. Overall, our framework not only achieves high correctness rate on target identification, but also finishes the task in much shorter time.

\section{Acknowledgements}

This work is supported by the grant of Shenzhen municipal government for basic research on the basic technology of UAV swarm network (JCYJ20150629144717142).

\section{References}

[1] S. Waharte and N. Trigoni. (2010) Supporting search and rescue operations with UAVs. Proceedings of International Symposium on Robots and Security (ROBOSEC).

[2] E. Yanmaz and H. Guclu. (2010) Stationary and mobile target detection using mobile wireless sensor networks. Proceedings of the IEEE Conference on Computer Communications, pp. 1-5

[3] Wong E M, Bourgault F, Furukawa T. (2005) Multi-vehicle Bayesian Search for Multiple Lost Targets. IEEE International Conference on Robotics and Automation. IEEE Xplore,3169-3174.

[4] E. J. Forsmo, E. I. G, T. I. Fossen, and T. A. Johansen. (2013) Optimal search mission with unmanned aerial vehicles using mixed integer linear programming. Proceedings of IEEE Intl. Conference on Unmanned Aircraft Systems (ICUAS), pp. 253-259

[5] J. Hu, L. Xie, K.-Y. Lum, and J. Xu. (2013) Multiagent information fusion and cooperative control in target search. IEEE Transactions on Control Systems Technology, no. 99, pp. 1-13 
[6] Khan A, Yanmaz E, Rinner B. (2015) Information Exchange and Decision Making in Micro Aerial Vehicle Networks for Cooperative Search[J]. IEEE Transactions on Control of Network Systems,2(4):1-1.

[7] Jin Y, Liao Y, Minai A A, et al. (2005) Balancing search and target response in cooperative unmanned aerial vehicle (UAV) teams[J]. IEEE Transactions on Systems Man \& Cybernetics Part B Cybernetics, 36(3):571-587.

[8] Millet T, Casbeer D, Mercker T, et al. (2010) Multi-agent Decentralized Search of a Probability Map with Communication Constraints . AIAA Guidance, Navigation, and Control Conference.

[9] Riehl J R, Collins G E, Hespanha J P. (2011) Cooperative Search by UAV Teams: A Model Predictive Approach using Dynamic Graphs[J]. Oct. IEEE Transactions on Aerospace \& Electronic Systems,47(4):26372656.

[10] George J, P. B. S, Sousa J B. (2011) Search Strategies for Multiple UAV Search and Destroy Missions.[J]. Journal of Intelligent \& Robotic Systems,61(1):355-367.

[11] Waharte S, Trigoni N, Julier S. (2009) Coordinated Search with a Swarm of UAVs. Sensor, Mesh and Ad Hoc Communications and Networks Workshops. SECON Workshops '09. IEEE Communications Society Conference on. IEEE Xplore.

[12] Chung T H, Burdick J W. (2008) Multi-agent Probabilistic Search in a Sequential Decision-theoretic Framework[C] . IEEE International Conference on Robotics and Automation. IEEE Xplore, 146-151.

[13] T. Furukawa, F. Bourgault, B. Lavis, and H. F. Durrant-Whyte. (2006) Recursive Bayesian Search-andTracking Using coordinated UAVs for Lost Targets. In Proceedings of IEEE Intl. Conference on Robotics and Automation, pp. 2521-2526.

[14] T. Chung and J. Burdick, (2008) Multi-agent probabilistic search in a sequential decision-theoretic framework. In Robotics and Automation. ICRA 2008. IEEE International Conference on, pp. 146-151.

[15] Happe J, Berger J.(2010) CoUAV: a multi-UAV cooperative search path planning simulation environment $[C]$. Summer Computer Simulation Conference. Society for Computer Simulation International, 8693.

[16] F. Bourgault, T. Furukawa, and H. F. Durrant-Whyte.(2003) Coordinated Decentralized Search for a Lost Target in a Bayesian World. In Proceedings of the IEEE/RSJ Intl. Conference on Intelligent Robots and Systems, 2003, pp. 48-53.

[17] F. Bourgault, T. Furukawa, and H. Durrant-Whyte.(2004) Decentralized Bayesian negotiation for cooperative search.In Proceedings of IEEE/RSJ Intl. Conference on Intelligent Robots and Systems, pp. 2681-2686.

[18] T. Furukawa, F. Bourgault, B. Lavis, and H. F. Durrant-Whyte. (2006) Recursive Bayesian Search-andTracking Using coordinated UAVs for Lost Targets. In Proceedings of IEEE Intl. Conference on Robotics and Automation, pp. 2521-2526. 